\title{
Dysregulated claudin-5 cycling in the inner retina causes retinal pigment epithelial cell atrophy
}

\author{
Natalie Hudson, ${ }^{1}$ Lucia Celkova, ${ }^{1}$ Alan Hopkins, ${ }^{1,2}$ Chris Greene, ${ }^{1}$ Federica Storti, ${ }^{3}$ Ema Ozaki, ${ }^{4}$ \\ Erin Fahey, ${ }^{4}$ Sofia Theodoropoulou, ${ }^{5}$ Paul F. Kenna, ${ }^{1,2}$ Marian M. Humphries, ${ }^{1}$ Annie M. Curtis, ${ }^{6}$ \\ Eleanor Demmons, ${ }^{7}$ Akeem Browne, ${ }^{7}$ Shervin Liddie, ${ }^{7}$ Matthew S. Lawrence, ${ }^{7}$ Christian Grimm, ${ }^{3}$ \\ Mark T. Cahill, ${ }^{2}$ Pete Humphries, ${ }^{1}$ Sarah L. Doyle, ${ }^{4}$ and Matthew Campbell ${ }^{1}$ \\ ${ }^{1}$ Smurfit Institute of Genetics, Trinity College Dublin, Dublin, Ireland. ${ }^{2}$ The Research Foundation, Royal Victoria Eye and

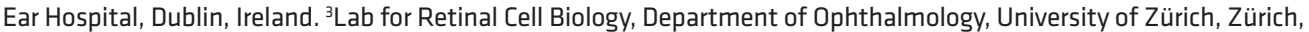 \\ Switzerland. ${ }^{4}$ School of Medicine, Trinity College Dublin, Dublin, Ireland. ${ }^{5}$ Academic Unit of Ophthalmology, School of \\ Clinical Sciences, University of Bristol, Bristol, United Kingdom. ${ }^{6}$ Department of Molecular and Cellular Therapeutics, Royal \\ College of Surgeons in Ireland, Dublin, Ireland. ${ }^{7} R x G e n$, Hamden, Connecticut, USA
}

Age-related macular degeneration (AMD) is the leading cause of central retinal vision loss worldwide, with an estimated 1 in 10 people over the age of 55 showing early signs of the condition. There are currently no forms of therapy available for the end stage of dry AMD, geographic atrophy (CA). Here, we show that the inner blood-retina barrier (BBRB) is highly dynamic and may play a contributory role in CA development. We have discovered that the gene CLDN5, which encodes claudin-5, a tight junction protein abundantly expressed at the iBRB, is regulated by BMAL1 and the circadian clock. Persistent suppression of claudin- 5 expression in mice exposed to a cholesterolenriched diet induced striking retinal pigment epithelium (RPE) cell atrophy, and persistent targeted suppression of claudin-5 in the macular region of nonhuman primates induced RPE cell atrophy. Moreover, fundus fluorescein angiography in human and nonhuman primate subjects showed increased retinal vascular permeability in the evening compared with the morning. These findings implicate an inner retina-derived component in the early pathophysiological changes observed in $A M D$, and we suggest that restoring the integrity of the iBRB may represent a novel therapeutic target for the prevention and treatment of GA secondary to dry AMD.

Conflict of interest: Trinity College Dublin owns an IP portfolio related to the regulation of the iBRB to prevent AMD.

Copyright: (c) 2019, American Society for Clinical Investigation.

Submitted: May 15, 2019

Accepted: June 27, 2019

Published: August 8, 2019.

Reference information: /CI Insight. 2019;4(15):e130273. https://doi. org/10.1172/jci.insight.130273.

\section{Introduction}

Age-related macular degeneration (AMD) is the leading cause of central retinal vision loss worldwide, and it has been estimated that $10 \%$ of people over the age of 55 already show early signs of disease. AMD presents in 2 forms that are generally referred to as either "dry" or "wet" AMD (1). Choroidal neovascularization $(\mathrm{CNV})$, associated with wet $\mathrm{AMD}$, occurs when blood vessels sprout from the underlying choroidal vasculature, disrupting the integrity of the retina and leading to acute vision loss. CNV occurs in approximately 1 in 10 AMD patients and can be treated with regular intraocular injection of antibodies or fusion proteins directed against VEGF (2). The end stage of "dry" AMD is termed geographic atrophy (GA), where the retinal pigment epithelium (RPE) progressively degenerates in the region of the macula and can eventually lead to death of cone photoreceptor cells and eventually central retinal vision loss (3). There is currently no therapeutic for GA other than recommended lifestyle changes, such as smoking cessation and dietary modification (4). Given the pervasive nature of AMD in the developed world and the wealth of research in this area, the underlying molecular pathology associated with GA development is still far from clear, with the majority of studies to date focused on understanding aberrant signaling and inflammatory mechanisms in the RPE.

The major early pathological hallmark common to both dry and wet AMD is the accumulation of drusen between the RPE and the choroid, with reticular pseudodrusen depositing in the subretinal space in some patients with late AMD and GA. Drusen is composed of extracellular components, including, but not limited to, amyloid- $\beta$, vitronectin, cholesterols, and almost every complement component (5). These components have all previously been identified in several tissues, including blood and photoreceptors, and 
it is most likely that AMD is primarily a condition that involves aberrant clearance mechanisms. Indeed, the source of extracellular materials in drusen has been characterized; the materials appear to be dominated by linoleate, implicating diet as the major contributor $(6,7)$.

Daily, RPE cells phagocytose POS that are shed during renewal of photoreceptors (8). Although some of the phagocytosed material is recycled to replenish essential components of the photoreceptors, other components in the material are exocytosed to the basolateral compartment of the RPE and are likely cleared by the systemic immune system via the choriocapillaris (9). This is a highly regulated process controlled by autophagic and phagocytic processes, and dysfunctional rates of clearance are likely a significant contributing factor to drusen accumulation in some individuals, as evidenced by residual body buildup in lysosomes observed in the RPE of AMD donor eyes $(10,11)$.

Although drusen deposition and localization differ from individual to individual, it is pertinent to consider that there is a considerable degree of symmetry in drusen patterning in each eye of a single individual $(12,13)$. This correlates with an equally high degree of interocular symmetry of retinal blood vessels between right and left eyes. These blood vessels, which form the inner blood-retina barrier (iBRB), are critical to maintaining retinal homeostasis $(14,15)$. Endothelial cells that line these vessels have evolved tight junctions, a series of up to 30 interacting proteins that limit the paracellular space between endothelial cells to all but the smallest of molecules. As well as regulating the exchange of ions and macromolecules between the blood and the delicate neural microenvironment, these highly specialized endothelial cells protect the retina by restricting the entry of potentially damaging blood-borne agents, such as neurotoxic chemicals, antibodies, pathogens, immune cells, and anaphylatoxins. They also express a variety of transporters to control both the selective transport of nutrients into the retina and the efflux of metabolites and toxins from the retina via the transcellular pathway (16-19).

Here, we provide evidence that one of the most enriched tight junction components at the iBRB, claudin-5 (20), is under the control of the transcription factor BMAL1 and the circadian clock. In addition, regulating its expression can very rapidly lead to an RPE atrophy phenotype in preclinical mouse and nonhuman primate models, and human subjects show evidence of a dynamic iBRB following fundus fluorescein angiography (FFA). Our data suggest that regulating the dynamic expression pattern of claudin-5 at the iBRB could control the burden of material consumed by the RPE daily. To our knowledge our findings represent the first description of $\mathrm{B} B \mathrm{~B}$ cycling with pathophysiological implications for the most common untreated forms of central retinal blindness.

\section{Results}

Claudin-5 expression at the iBRB is regulated by the circadian clock. Claudin-5 is the most enriched component of both the brain and retinal endothelial tight junctions (20). Indeed, its dose-dependent expression is critical, with knockout mice dying within hours of birth $(19,21)$. In neural tissues (cerebellum, hypothalamus, brainstem), we discovered that claudin-5 transcript levels varied depending on the time of day of tissue collection $(P=0.00051)$ (Figure 1A). Unusually, this phenomenon was observed in every tissue examined, with claudin-5 transcript levels being lower in the evening when compared with the morning in all tissues (Figure 1B). Indeed, when we specifically harvested retinas from mice every 4 hours over a 24-hour period, we observed a distinct phase of transcription mirroring all other tissues, where claudin-5 levels significantly decreased during a 12-hour period before increasing again (Figure 1C). This change in retinal claudin-5 expression was observed in the evening compared with the morning at both the protein and transcript level (Figure 1D, $P=$ 0.0064; and Figure 1E, $P=0.042$ ). These transcriptional differences in claudin-5 expression were confirmed as circadian as opposed to diurnal because dark-adapted (DA) mice showed the same pattern of expression (Figure 1F) as did mice that had their circadian rhythms inverted by altering their light cycle (Figure $1 \mathrm{G}$ ). Although other tight junction components ZO-1 and occludin appeared to cycle depending on the time of the day, these did not appear to be circadian in nature (Supplemental Figure 1; supplemental material available online with this article; https://doi.org/10.1172/jci.insight.130273DS1). Levels of claudin-5 were decreased in the microvasculature of the retina in the evening compared with the morning (Figure $1 \mathrm{H})$, and this was accompanied by the increased extravasation of a tracer molecule (EZ-Link-Biotin, 660-Da MW), with enhanced tracer signal observed in mice in the outer plexiform layer (OPL) and inner/outer segment regions of the retina in the evening compared with the morning (Figure 1 , I and J, $P=0.0184$ ).

Size-selective regulation of the $i B R B$. In mice examined using dynamic contrast-enhanced MRI (DCEMRI), enhanced gadolinium (800-Da MW) signal was observed in the retina in the evening when compared 
A

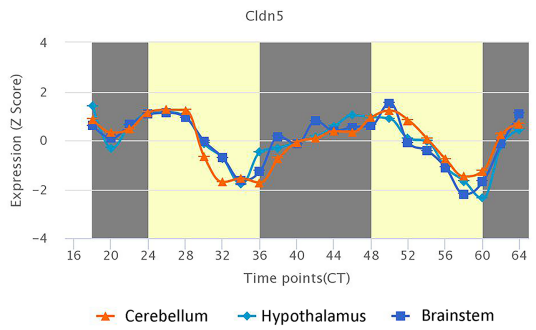

B

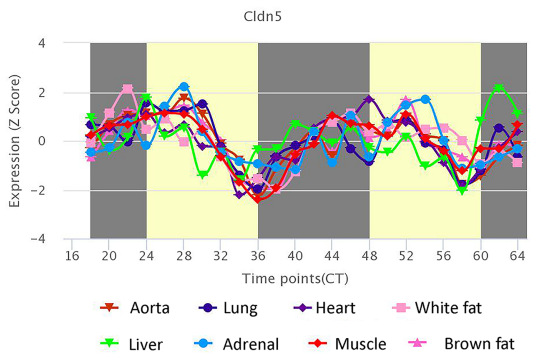

C

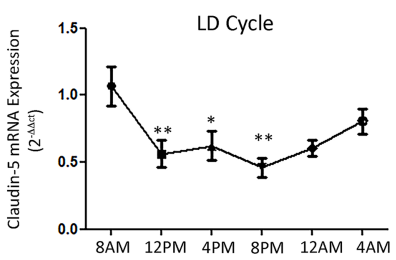

D

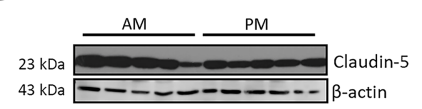

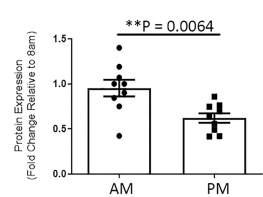

E

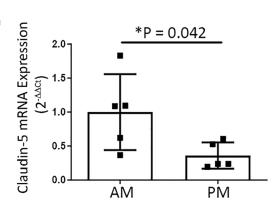

$\mathbf{F}$

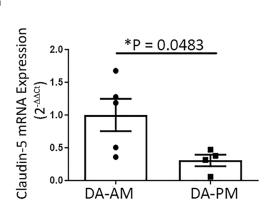

$\mathbf{G}$

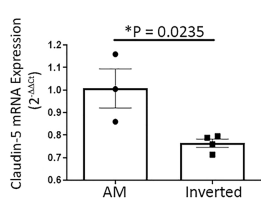

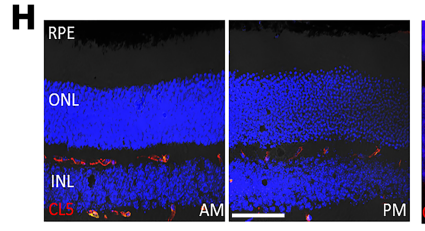

.

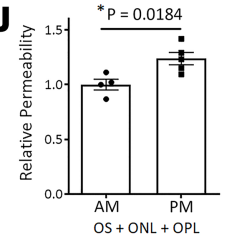

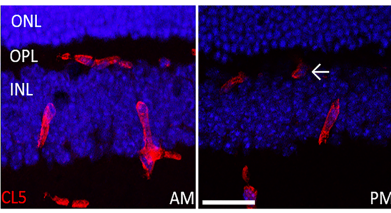

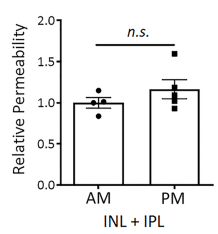

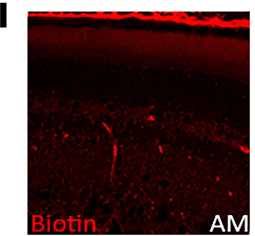

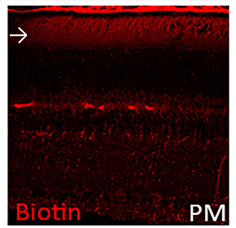

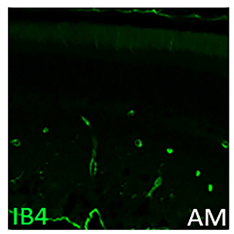

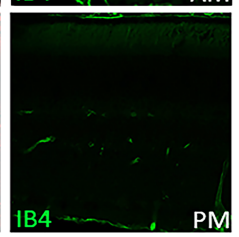

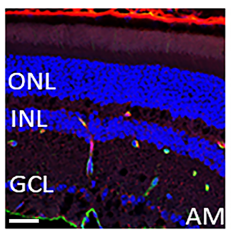
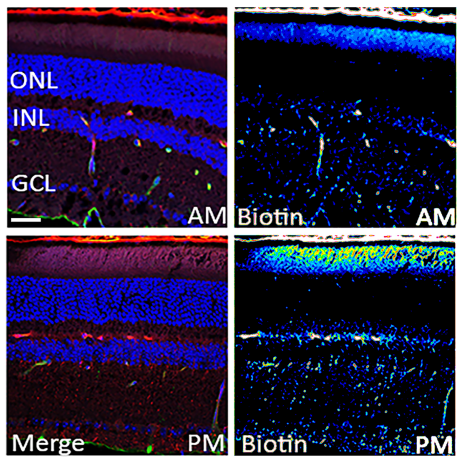

Figure 1. Claudin-5 cycling in the inner retina. (A) Claudin-5 transcript cycling in the mouse cerebellum, hypothalamus, and brainstem. (B) Claudin- 5 cycling in the mouse aorta, lung, heart, white fat, liver, adrenal gland, muscle, and brown fat $\left({ }^{*} P=0.00051\right.$ ), from data collated from open-source data available on http://cirgrdb.biols.ac.cn/. (C) Claudin-5 transcript cycling in the neural retina over a 24-hour period $\left({ }^{* *} P<0.01,{ }^{*} P<0.05\right.$, and $n=7-11$ mice). (D) Western blot analysis of claudin-5 expression at 8 am compared with 8 pm. Densitometric analysis (right, $\left.{ }^{* *} P=0.0064\right)$. $(n=5$ mice for each time point.) (E) qPCR analysis of claudin- 5 transcript at 8 am compared with $8 \mathrm{pm}$ ( ${ }^{*} P=0.042$, and $n=5$ mice for both time points). (F) Transcript levels at 8 am compared with $8 \mathrm{pm}$ in mice dark adapted (DA) for 24 hours ( ${ }^{*} P=0.0483, n=5$ mice for DA-AM, and $n=4$ mice for DA-PM). (G) Levels of claudin-5 transcript in mice with inverted circadian rhythm ( ${ }^{*} P=0.0235, n=3$ mice for morning, and $n=4$ mice for inverted). (H) Claudin-5 (red) levels in the retinas of mice at $8 \mathrm{am}$ (left) compared with 8 pm (right). ONL, outer nuclear layer; INL, inner nuclear layer; GCL, ganglion cell layer; scale bar: $100 \mu$ m. High-magnification images of claudin-5 (red) in the retinas of mice at 8 am (left) compared with 8 pm (right); scale bar: $50 \mu \mathrm{m}$. (I) EZ-Link-Biotin (red) extravasation in the inner and outer segments of the photoreceptors at $8 \mathrm{pm}$ compared with $8 \mathrm{am}$. Retinal vasculature stained with isolectin IB4 (green) scale bar: $50 \mu \mathrm{m}$. (J) Significant extravasation of EZ-Link-Biotin in the outer segments, ONL, and OPL (* $P=0.0184)$. No significant signal observed in the INL, inner plexiform layer (IPL), or GCL. Student's $t$ test, with significance represented by a $P$ value of less than or equal to 0.05 . For multiple comparisons, ANOVA was used with Bonferroni's post hoc test and significance represented by a $P$ value of less than or equal to 0.05 .

with the morning (Figure 2A, $P<0.001$ ), and this was also manifested when fundus fluorescein angiograms (FFAs) were conducted, with enhanced sodium fluorescein (376-Da MW) signal observed in the deeper vascular plexus in the evening when compared with the morning (Figure $2 \mathrm{~B}, P<0.001$ ). This was also observed in other strains of mice, including 129SV and CD1 mice (Supplemental Figure 2). Intriguingly, this circadian change in permeability appeared to be size selective because no differences were observed in FFA analysis using an FITC-labeled dextran molecule of $4 \mathrm{kDa}$ (FITC-Dextran) (Figure 2C), suggesting claudin-5 cycling at the $\mathrm{iBRB}$ is regulating the passive diffusion of molecules below this molecular weight.

Bmal-1 regulates claudin-5 levels in mouse and human retinal endothelial cells. Bmal-1 is a transcription factor that is an essential component of the circadian clock (22). In mice with a targeted disruption of Bmal-1 expression in the vascular endothelium (Bmallflffl Tie2-Cre), levels of claudin-5 protein remained unchanged in the retinas of mice between the morning and evening (Figure 3A), and this effect was observed at the transcript level even in mice lacking only a single Bmall allele in the retinal endothelium (Figure 3B). The levels and pattern of expression of claudin-5 appeared the same in retinas of Bmal1 ${ }^{f l f l}$ Tie2-Cre $e^{+}$mice (Figure 3C), and there was no difference between FFAs performed in these mice in the morning and evening (Figure 3, D and E). This phenotype was also observed in mice with a single Bmal1 allele remaining, namely Bmal1 ${ }^{\text {WT/fl }}$ Tie2-Cre (Supplemental Figure 3). 
A
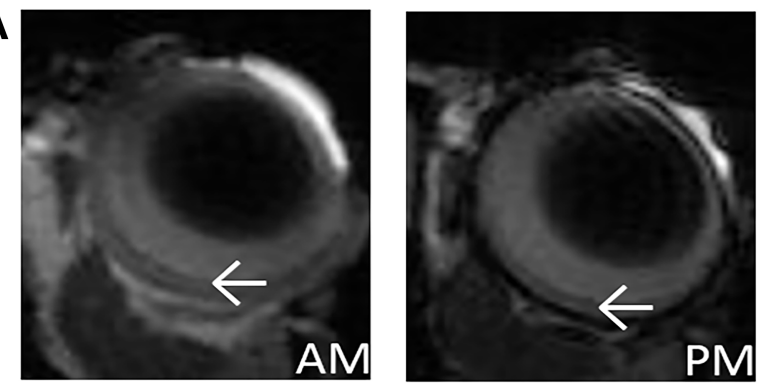

B
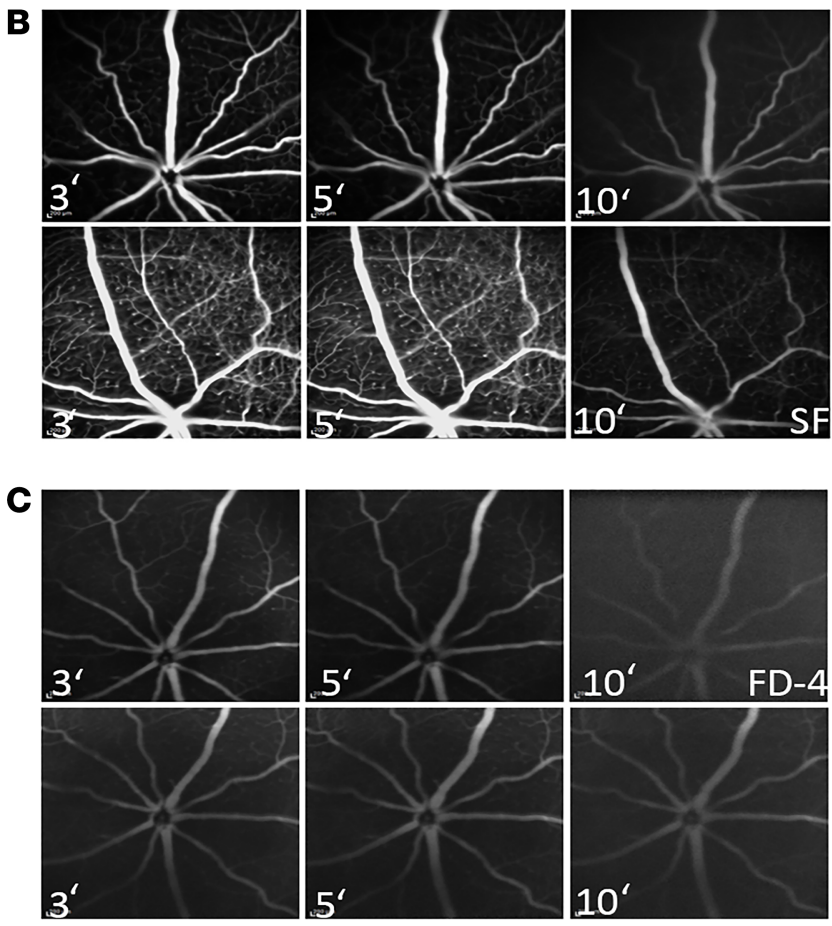

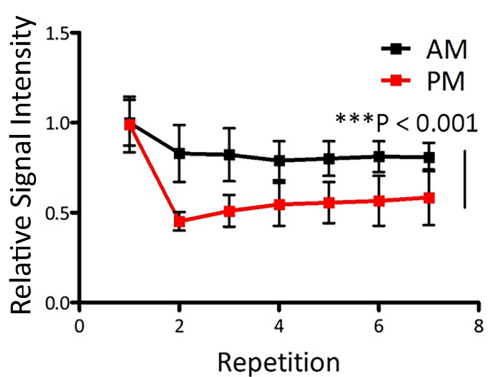

AM

PM

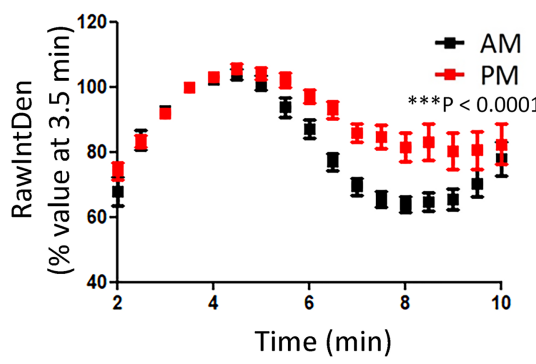

AM

PM

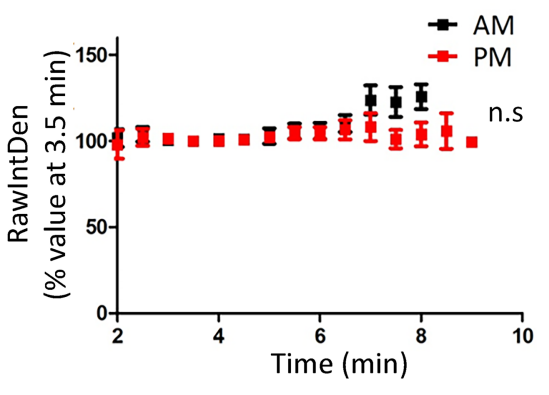

Figure 2. iBRB integrity in the morning compared to the evening in mice. (A) Gadolinium (800-Da MW) extravasation in the retina of mice at 8 pm (right) compared with $8 \mathrm{am}$ (left). Dynamic contrast-enhanced MRI (DCE-MRI) is quantified in histogram at right. ${ }^{* * *} P<0.001$ ( $n=5$ mice for 8 am, and $n=4$ mice for 8 pm). (B) Fundus fluorescein angiography (FFA) 3, 5, and 10 minutes after sodium fluorescein (MW: 376 Da) injection at 8 am (top) compared with $8 \mathrm{pm}$ (bottom), with enhanced signal at 8 pm (*** $P<0.001$, and $n=12$ mice for both time points). (C) FFA analysis of FITC-Dextran-4 (FD4) (MW: $4000 \mathrm{Da}$ ) at $8 \mathrm{am}$ (top) compared with $8 \mathrm{pm}$ (bottom) ( $n=8$ mice for both time points). ANOVA was used with Bonferroni's post hoc test and significance represented by a $P$ value of less than or equal to 0.05 .

Cells in vitro do not have an entrained circadian rhythm; however, rhythmicity can be induced by serum shocking cells with 50\% serum for 2 hours (23). Endothelial cells were exposed to a serum shock, and claudin-5 levels were observed to cycle in a manner identical to that observed in mice (Figure $3 \mathrm{~F}$ ). RNAi-mediated suppression of the expression of BMAL1 in endothelial cells before serum shock, however, resulted in a transient cessation of claudin-5 cycling (Figure 3, G and $\mathrm{H}$ ) that was accompanied by a decrease in transendothelial electrical resistance (TEER) and a discontinuous pattern of expression at the plasma membrane of cells (Figure 3, I and J).

Targeted suppression of claudin-5 with a high-cholesterol diet induces RPE atrophy. To recapitulate a persistent phenotype similar to that observed in WT mice in the evening (i.e., high sodium fluorescein signal), we subretinally injected an adeno-associated virus (AAV9) vector expressing claudin-5 shRNA under the control of a doxycycline-inducible promoter. A nontargeting (NT) AAV9 vector expressing doxycycline-inducible shRNA targeting the nonmammalian gene luciferase was used as a control. It is well established that environmental factors, such as smoking and a high-fat/high-cholesterol diet, are major risk factors for AMD; in that regard, mice were fed a high-cholesterol diet (HCD) as described previously (24), and doxycycline was supplemented in the drinking water. Within 3 weeks of constant claudin-5 suppression, enhanced sodium fluorescein extravasation was observed in mice fed a normal 
A

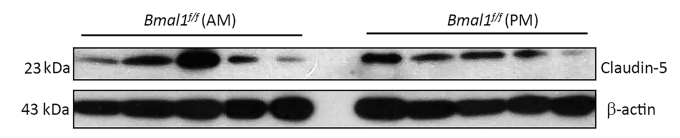

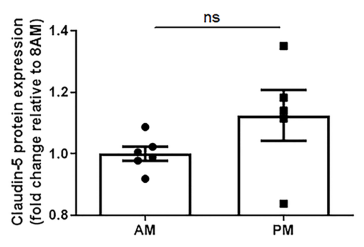

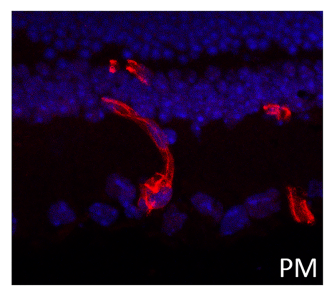

B

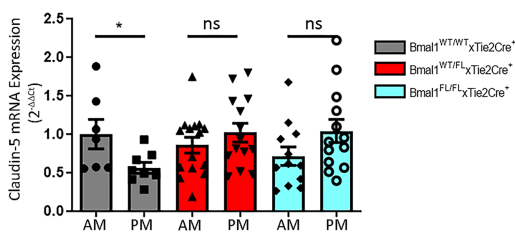

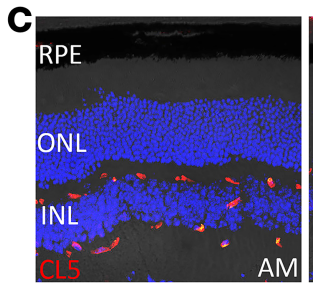
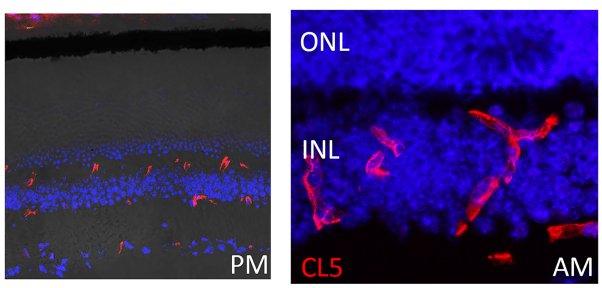

D

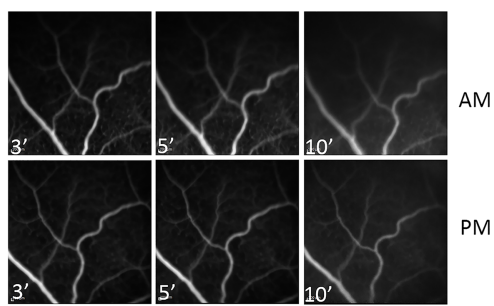

E

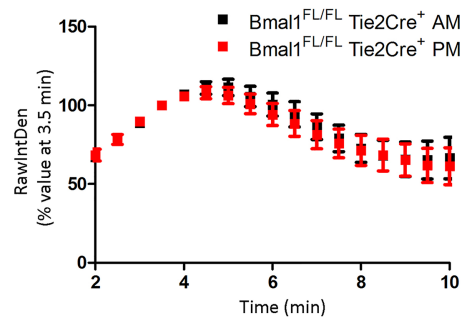

$\mathbf{F}$

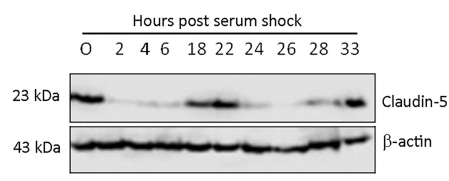

G

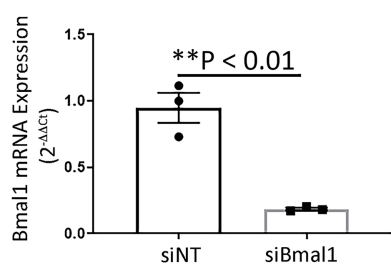

H

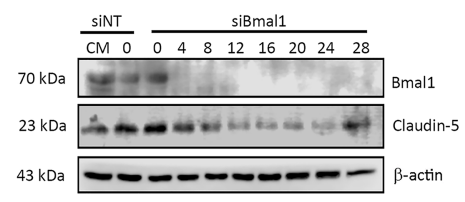

I

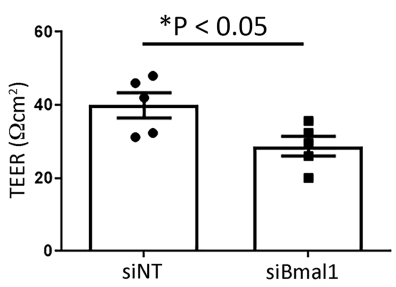

J

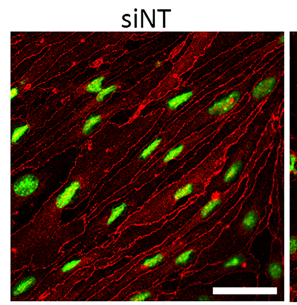

siBmal1

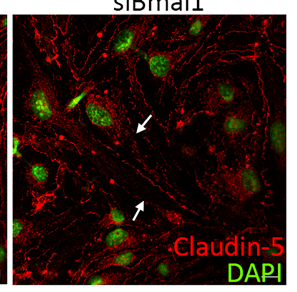

Figure 3. Bmal1 regulates claudin-5 levels at the iBRB. (A) Claudin- 5 expression at 8 am and $8 \mathrm{pm}$ in Bmalf/f/fl Tie2-Cre+ mice at 8 am compared to 8 pm ( $n=5$ mice for both time points, $P=n s)$. (B) Claudin-5 transcript levels in WT Cre ${ }^{+}, B$ mal/ ${ }^{W T / f l}$ Tie2-Cre ${ }^{+}$, and Bmalf $f^{f / f l}$ Tiez-Cre $^{+}$mice $(n=7-13$ mice per group). (C) Claudin-5 expression in retinas of Bmal/7/ff Tie2-Cre+ at 8 am (left) compared with 8 pm (right), higher magnification. Original magnification, $\times 40$. (D) FFA in Bmalf f//f Tiez-Cre at 8 am (top) compared to 8 pm (bottom) showed (E) no significant differences $(n=12$ mice for 8 am, and $n=11$ mice for 8 pm). (F) Claudin-5 expression in endothelial cells exposed to serum shock (50\% serum) for 2 hours. (G) Suppression efficacy of Bmal-1 siRNA on Bmal-1 transcript expression ( ${ }^{*} P=0.0026$, and $n=3$ ). (H) Claudin-5 expression in bEnd.3 cells with suppressed Bmal1 before serum shock exposure. (I) Transendothelial electrical resistance (TEER) measurement in bEnd. 3 cells with Bmal1 suppressed ( ${ }^{*} P=0.0339$, and $n=5$ ). (J) Claudin-5 staining in bEnd.3 cells after suppression of Bmal1. Red, claudin-5; green, nuclei. Scale bar: $50 \mu \mathrm{m}$. Arrows indicate discontinuity of claudin- 5 staining. Student's $t$ test, with significance represented by a $P$ value of less than or equal to 0.05 . For multiple comparisons, ANOVA was used with Bonferroni's post hoc test and significance represented by a $P$ value of less than or equal to 0.05 .

diet (Figure 4A, top). However, in mice with suppressed claudin-5 fed an HCD, enhanced fluorescein signal was observed in the deeper vascular plexus with apparent hyperreflectivity of the underlying RPE (Figure 4A, bottom). Analysis of histological sections of the retina showed enlarged subretinal cells that contained a large amount of autofluorescent material (Figure 4B). Depigmented RPE cells and in some areas a lack of RPE cells was evident in semithin sections of retinas from these mice (Figure 4C). Analysis of flat mounts from eyes of these mice stained for the tight junction component ZO-1 showed regions of atrophic RPE where the underlying choroid was visible (Figure 4D). Because claudin-5 is not expressed in the RPE, and the AAV will transduce endothelial cells only within the retinal side of the outer blood-retina barrier, these data are strongly suggestive of the passive paracellular diffusion of material from the systemic circulation toward the RPE (Figure 4E). To suppress claudin- 5 in both the retina and the choriocapillaris, we developed a mouse model that would allow us to induce suppression of claudin-5 throughout the whole body (Figure 4, F and G, and ref. 21). A similar phenotype was observed in these mice with a targeted "knockin" of claudin- 5 shRNA, with enlarged and vesiculated RPE cells observed (Figure $4 \mathrm{H}$ ) in mice with decreased levels of claudin-5 fed a cholesterol-rich diet. These mice died following chronic suppression of claudin-5, so we could not follow them up at later time points. 
A

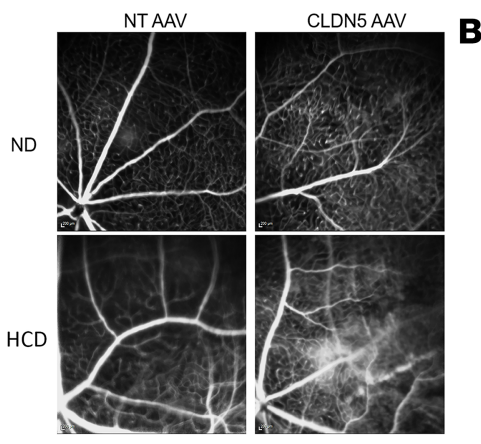

D

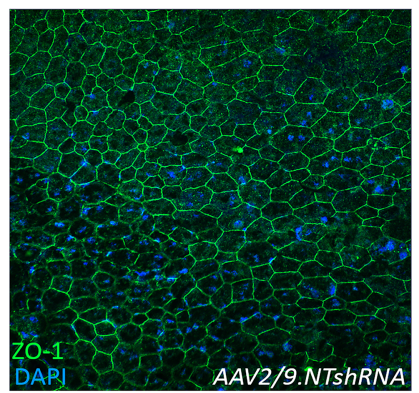

B

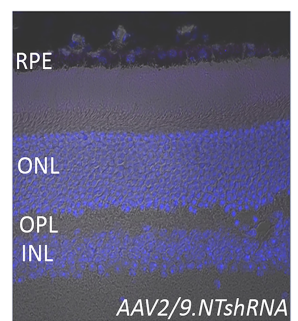

C

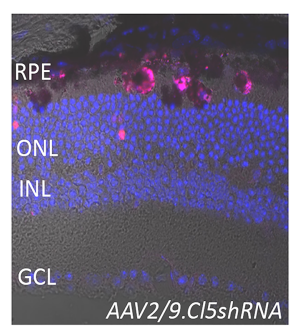

AAV2/9.NTshRNA

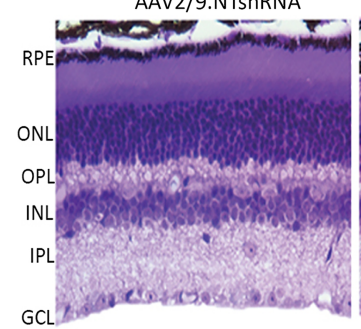

AAV2/9.CI5ShRNA

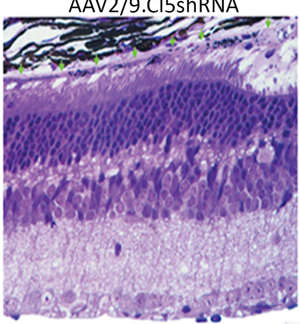

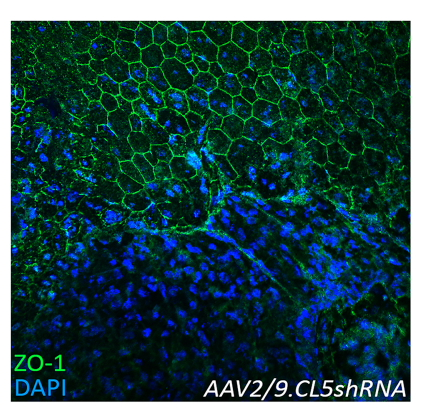

E

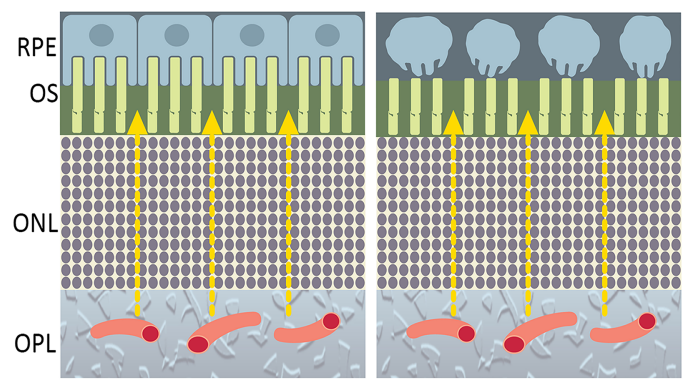

$\mathbf{F}$

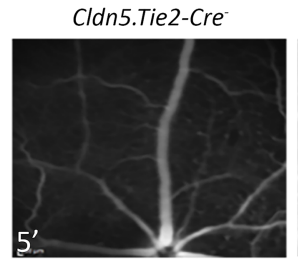

Cldn5.Tie2-Cre
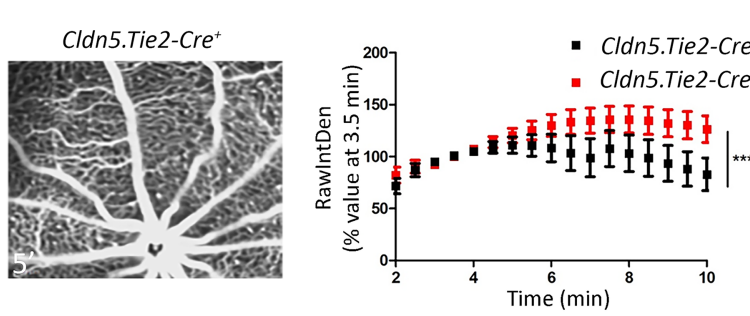

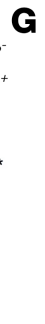

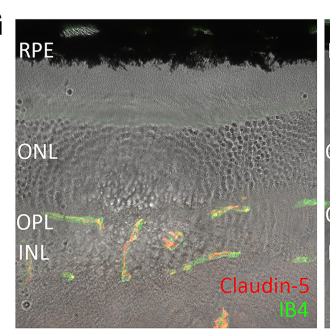

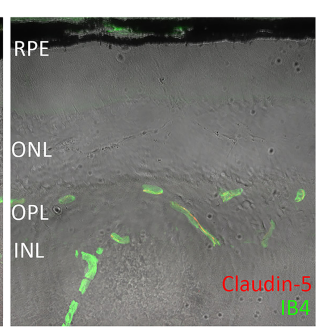

H
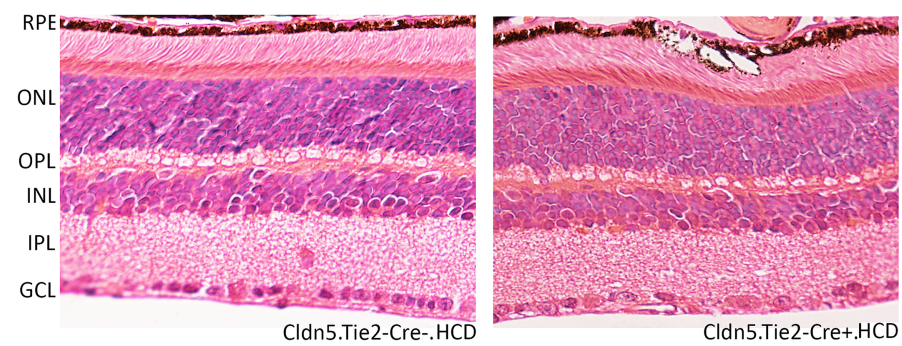

Figure 4. Persistent claudin-5 suppression induces RPE cell atrophy. (A) FFA in mice injected with an NT shRNA AAV2/9 and fed a normal diet (ND) (top left), or HCD (bottom left). FFA analysis of mice injected subretinally with a Cldn5-targeting shRNA AAV2/9 and fed an ND (top right) or an HCD (bottom right) ( $n=$ 12 mice per group). (B) Retinal histology of mouse injected subretinally with an NT shRNA AAV2/9 and fed an HCD (left) or claudin-5-targeting shRNA AAV2/9 and fed an HCD (right). Blue, DAPI; red, autofluorescence. (C) Semithin sections of mice injected with NT AAV or claudin-5 AAV and fed an HCD. (D) ZO-1 (green) staining in flat mounts of mice receiving AAV2/9.NTshRNA subretinally (left) or AAV2/9.CL5shRNA (right). (Images representative of $n=12$ mice per group.) (E) Schematic representation of retinal interstitial kinesis. Normal paracellular flux of blood-derived components toward the RPE (left). Aberrant flux of material from the inner retina inducing RPE atrophy. (F) FFA analysis in Cldn5 Tiez-Cre- mice (left). FFA analysis in Cldn5 Tie2-Cre mice (right). Quantitative analysis of fluorescein leakage in Cldn5 Tiez-Cre+ mice $\left({ }^{* *} P<0.001, n=7\right.$ mice for Cldn5 Tie2-Cre-, and $n=8$ mice for Cldn5 Tie-Cre $)$. (C) Claudin-5 (red) expression in Cldn5 Tie2-Cre- mice (left) compared with Cldn5.Tie2-Cre+ mice (right). Green, IB4. (H) Retinal histology in Cldn5 Tie2-Cre- mice fed an HCD (left) compared with Cldn5 Tie2-Cre ${ }^{+}$mice fed an HCD (right). ( $n=7$ mice.) Blue, DAPI. Student's $t$ test was used, with significance represented by a $P$ value of less than 0.05 . ANOVA was used with Bonferroni's post hoc test and significance represented by a $P$ value of less than or equal to 0.05 . Original magnification, $\times 40$ (B-D, G, and $\mathbf{H}$ ).

The human and nonhuman primate macula is more permeable in the evening, and suppression of claudin-5 in the macula can induce RPE atrophy. To identify the regional expression of claudin-5 in the human eye, we examined CLDN5 transcript expression in the central retina (which includes the macula but also the parafovea; the punch from which the RNA was isolated was about $5 \mathrm{~mm}$ in diameter) and the midperipheral retina ( $n=28$ donor eyes). Claudin-5 levels were significantly increased in the central macula compared with the midperipheral retina $(P<0.0001)$ (Figure 5A). To determine the integrity of the iBRB in human subjects in the morning compared with the evening, we recruited 15 healthy participants (aged between 18 and 30 years old) to undergo FFA analysis at a defined time in the morning and again in the evening with 
A

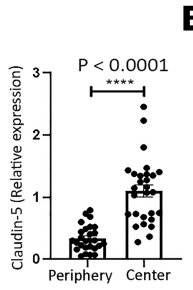

B

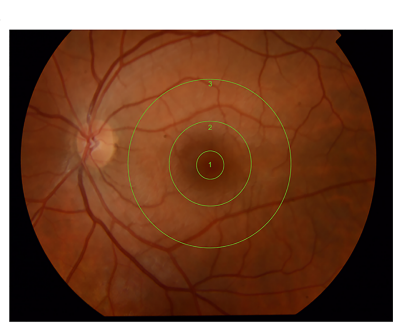

$\mathbf{E}$
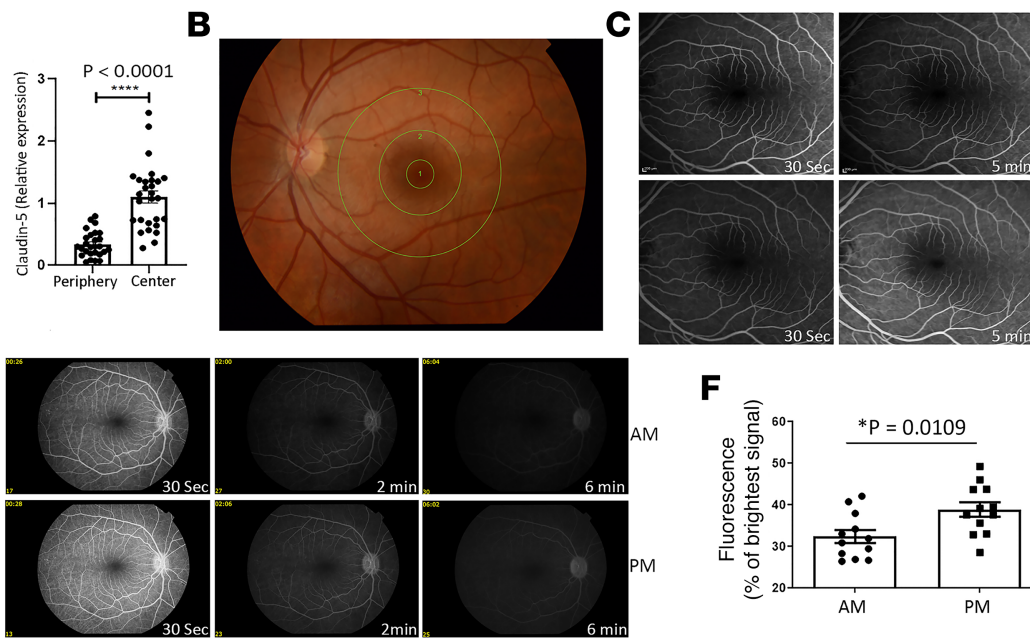

$\mathbf{F}$

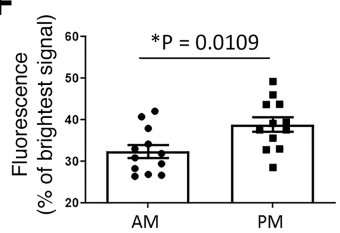

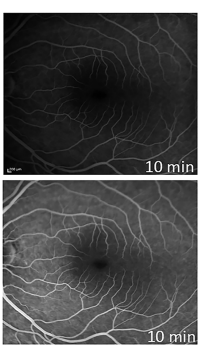
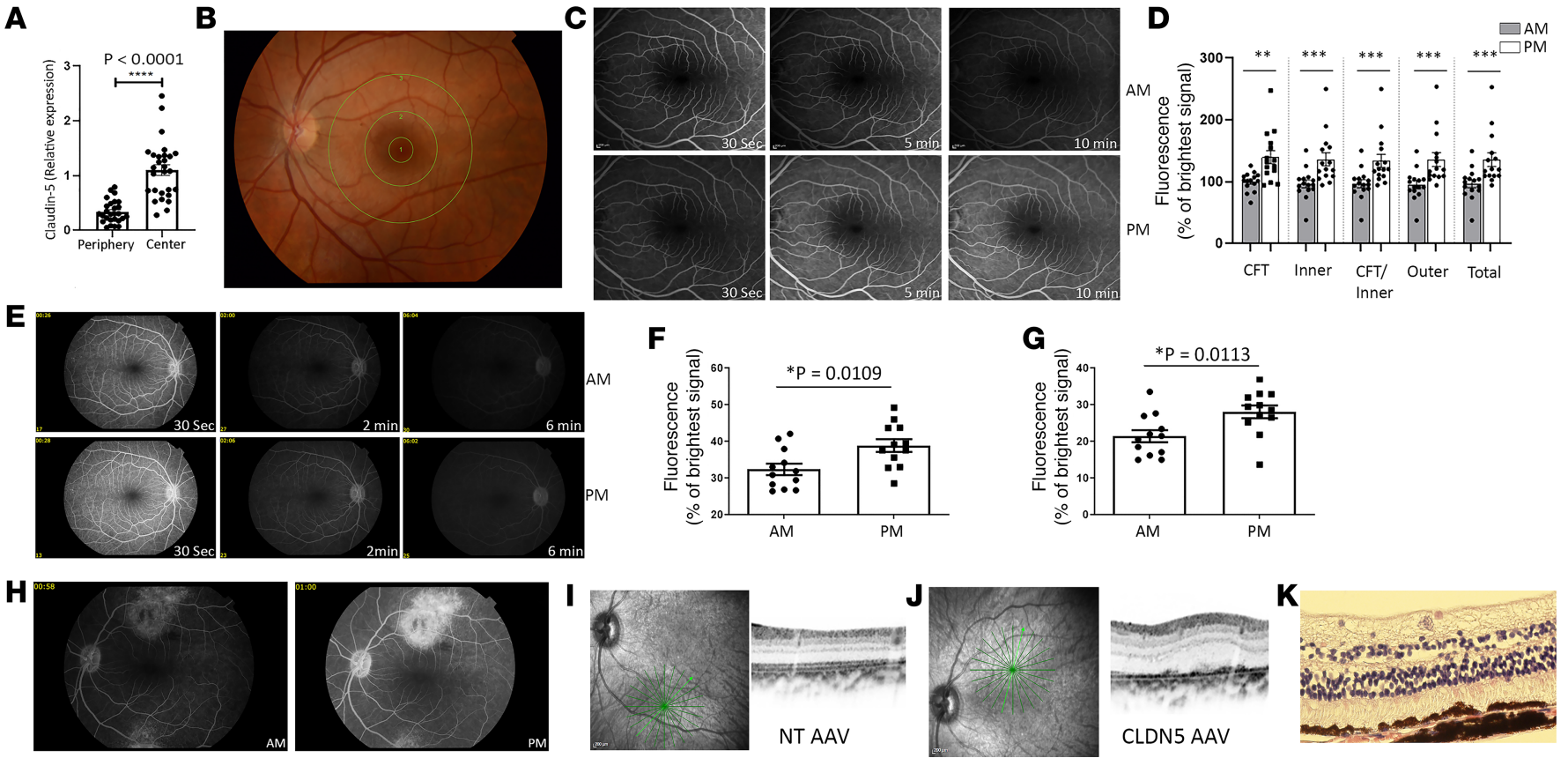

Figure 5. Regulation of the iBRB in humans and nonhuman primates. (A) Claudin-5 expression in the central human retina compared with the peripheral retina ( $n=28$ donor human eyes, and ${ }^{* *} P<0.0001$ ). (B) ETDRS grid over the human macula. (C) FFA in the human retina 30 seconds, 5 minutes, and 10 minutes after i.v. sodium fluorescein injection (top, morning; bottom, evening). (D) Significantly increased fluorescein signal in evening compared with morning in the central fovea (CFT) ${ }^{* *} P<0.01$ ), inner macula ( $\left.{ }^{* *} P<0.001\right)$, CFT and inner macula $\left({ }^{* *} P<0.001\right)$, outer macula ( $\left.{ }^{* * *} P<0.001\right)$, and total area combined $\left({ }^{* *} P<0.001\right)$. (E) FFA analysis from the nonhuman primate (top, morning; bottom, evening). (F) Individual fluorescence signal in each monkey at morning compared with evening at 2 minutes $\left({ }^{*} P=0.0109\right)$ and $(\mathbf{C}) 6$ minutes $\left({ }^{*} P=0.0113\right)$ after i.v. injection $(n=6$ monkeys, 12 eyes). (H) FFA analysis of retina injected subretinally with claudin-5-targeting shRNA AAV in the superior macula and NT AAV in the inferior retina and FFA analysis performed in the morning (left) and evening (right). ( $n=3$ monkeys.) (I) OCT analysis of the NT AAV-injected and (J) claudin-5 shRNA AAV-injected region. (K) Histological analysis of retina injected with AAV2/9 expressing claudin-5-targeting shRNA ( $n=3$ monkeys, 6 eyes). Original magnification, $\times 40$. Student's $t$ test, with significance represented by a $P$ value of less than or equal to 0.05 .

a minimum of 60 hours between visits. The chronotype of each person was determined using the Munich Chronotype Questionnaire (Supplemental Table 1). For analysis, the macula was divided into 3 regions based on the Early Treatment Diabetic Retinopathy Study (ETDRS) grid, comprising (a) central fovea, (b) inner macula, and (c) outer macula (Figure 5B). Fluorescein signal was evident and more prolonged in the evening compared with the morning in the same subject (Figure 5, C and D), and this was significantly increased in all macular regions analyzed ( $n=15$ subjects, and $P<0.001)$. The phenotype was also observed in nonhuman primates, whereby increased fluorescein signal was observed in the macula in the evening compared with the morning ( $n=6$ monkeys, 12 eyes) (Figure 5, E-G, $P<0.05$ ).

Although the use of mouse models of disease has added greatly to our understanding of human conditions, it must be recognized that mice lack a cone-rich macula similar to the human retina. Therefore, we designed an AAV expressing a doxycycline-inducible shRNA targeting a homologous region of the human and African green monkey claudin-5 mRNA. Three monkeys were injected subretinally superiorly in the macular region with an AAV targeting claudin-5 or inferiorally with an NT AAV. Monkeys were supplemented with doxycycline in their diet for 3 weeks after injection, and FFA analysis showed clear fluorescein extravasation at the site of claudin-5 AAV injection (superior macula) compared with the NT AAV (inferior macula) (Figure $5 \mathrm{H})$. Optical coherence tomography (OCT) analysis of the region of injection showed normal laminar structure in NT AAV-injected regions (Figure 5I); however, aberrant RPE structure was clearly evident at the claudin-5 AAV-injected site (Figure 5J). This phenotype was confirmed by histological analysis of retinal cryosections of this region (Figure $5 \mathrm{~K}$ ).

\section{Discussion}

Here, we describe for the first time to our knowledge an inner retina-derived contribution to RPE cell atrophy. Our series of observations could have major implications for the prevention of AMD in general and more specifically of GA secondary to dry AMD. 
We have shown that the major iBRB-associated tight junction component, claudin-5, cycles in a distinct circadian rhythm and is regulated by the circadian clock component BMAL1. Claudin-5 is one of the central mediators of paracellular passive diffusion of material from blood to retina and vice versa and appears to be a critical factor in allowing for the daily passive diffusion of dietary material from the blood to the retina $(13,14)$. In this regard, we have shown that size-selective passive diffusion of systemically injected tracer molecules will move from the inner retinal vasculature into the retinal parenchyma and diffuse toward the outer retina and RPE in mice, nonhuman primates, and human subjects. This is important because an inner retina-derived contributor to RPE pathology has not been described previously to our knowledge and may represent a critically important and early physiological process central to the development of AMD.

We have shown that chemical and genetic ablation of Bmal-1 function can prevent claudin-5 cycling and affect the integrity of endothelial cells when RNAi-mediated suppression of Bmal-1 transcripts is induced. RNAi-based experiments highlighted the importance of Bmal-1 turnover in regulating claudin-5 expression because knockout of Bmal1 from endothelial cells prevented its cycling in vivo but decreased the levels of claudin-5 overall in RNAi-based experiments. It is of note that BMAL1-mediated circadian rhythmicity is dysregulated in the aged population (25-27), and it is tempting to suggest aberrant claudin-5 cycling in the human retina with age is a contributing factor to drusen accumulation and subsequent RPE atrophy.

It is well known that poor diet and smoking are major risk factors for AMD (28). Here, we found that a cholesterol-enriched diet in tandem with low levels of claudin-5 expression were enough to rapidly produce a pathological RPE, suggesting that individuals with suppressed cycling of claudin-5 may exacerbate RPE pathology with poor dietary intake.

For the treatment of CNV secondary to wet AMD and GA secondary to dry AMD, retinal translocation surgical procedures were previously undertaken in an effort to restore visual acuity in patients by surgically translocating the healthy neural retina from the atrophic RPE-affected area to a region of intact RPE cells. Although these techniques represented advanced and technically demanding surgeries, they proved successful for a subgroup of patients with subfoveal CNV. It was of note, however, that up to $47 \%$ of patients exhibited RPE atrophy in the translocated region within 45 months of surgery, suggesting an inner retina-derived process that could promote RPE cell stress and death $(29,30)$. Interestingly, iBRB breakdown has been related to a rare juvenile retinal condition, Coats' disease, where leakage of blood-derived cholesterol crystals and lipid-laden macrophages infiltrate and fill the subretinal space. The pathophysiology of this condition lends weight to an inner retinal vascular-derived deposition of material toward the RPE (31). It must also be noted that endothelial cells become hyperpermeable in conditions such as diabetic retinopathy, independent of any overt RPE atrophy. We postulate, however, that chronic and size-selective disruption of the $\mathrm{iBRB}$ will lead to the accumulation of material in the RPE over a prolonged period. We therefore propose a circadian-entrained cycling of the permeability of the inner retinal vasculature as a key contributing factor to a dynamic retinal interstitial kinesis that allows for replenishment of substrate to the photoreceptors and the clearance of material from the neural retina daily. We propose that in the aging eye, with dysregulation of the circadian clock, this interstitial process ceases to be tightly regulated and therefore will overload the RPE with spent metabolites and dietary components that will lead to drusen deposition and eventual RPE atrophy. Although our data presented cannot yet speak to the cycling of claudin-5 at the iBRB in the aging eye, ongoing studies aimed at elucidating changes in retinal permeability in preclinical species and AMD patients will extend our observations.

Overall, our findings directly implicate claudin-5 as a key mediator of subsequent RPE pathology and suggest that methods aimed at restoring the dynamic expression of this molecule in the aging eye may prevent or indeed halt the progression of AMD.

\section{Methods}

Animal experiments and experimental groups. All studies carried out in the Smurfit Institute of Genetics in Trinity College Dublin (TCD) adhered to the principles laid out by the internal ethics committee at TCD, and all relevant national licenses were obtained before commencement of all studies. C57BL/6J mice were sourced from Jackson Laboratory and bred on-site at the Smurfit Institute of Genetics in TCD. Before experiments, all mice were kept on a 12-hour light/12-hour dark cycle with lights on at 8 am and lights off at $8 \mathrm{pm}$. For experiments involving inversed cycles, mice were kept on a 12-hour light/12-hour dark cycle with lights on at $8 \mathrm{pm}$ and lights off at 8 am for a minimum of 3 weeks. 
Hematoxylin and eosin staining. Paraffin sections were deparaffinized by dipping 10 times in xylene followed by rehydration in 10 dips each of $100 \%, 90 \%$, and $70 \%$ ethanol. Slides were incubated in hematoxylin solution for 6 minutes, rinsed in water, and then incubated in eosin solution for 2 minutes. Slides were rinsed in water and dehydrated by dipping 10 times in $70 \%, 90 \%$, and $100 \%$ ethanol and once in xylene. Coverslips were placed on slides using Sub-X Mounting Medium (Leica Biosystems). Slides were analyzed under a light microscope (Olympus 1X81).

Indirect immunostaining of retinal flat mounts and retinal cryosections. Mouse retinal cryosections (12- $\mu \mathrm{m}$ thick) were fixed with methanol for 10 minutes at $-20^{\circ} \mathrm{C}$, washed twice in PBS, and blocked/permeabilized with $5 \%$ normal goat serum (NGS) and 0.5\% Triton X-100 in $1 \times$ PBS for 30 minutes at room temperature. Sections were incubated with primary antibodies (rabbit anti-mouse claudin-5, Invitrogen, 34-1600, and rabbit antimouse ZO-1, Invitrogen, 40-2200) at $4^{\circ} \mathrm{C}$ overnight. Sections were washed 3 times for 5 minutes each in PBS and incubated with fluorescent-labeled secondary antibodies (goat anti-rabbit Cy3, Abcam, ab6939, and goat anti-rabbit Cy2, Abcam, ab150077) (1:500) for 2 hours at room temperature followed by three 5-minute washes with PBS. To label blood vessels, sections were costained with Isolectin-IB4 Alexa Fluor 488 (Invitrogen, I21411) (1:300). All sections were counterstained with Hoechst 33258 (MilliporeSigma, B2261) (1:10,000) for 30 seconds and mounted with Aqua Poly/Mount (Polysciences, Inc). Images were acquired using a Zeiss LSM 710 confocal laser scanning microscope. Identical acquisition settings were used to acquire images, and image processing was performed in Adobe Photoshop and ImageJ (NIH).

Western blot analysis. Retinal tissue and cells were lysed in RIPA lysis buffer with phosphatase and protease inhibitors (MilliporeSigma), incubated on ice for 10 minutes, and centrifuged at $12,000 \mathrm{~g}$ at $4^{\circ} \mathrm{C}$ for 20 minutes. Ten micrograms of protein was resolved on $12 \%$ SDS polyacrylamide gels. Proteins were transferred to PVDF membranes and blocked for 1 hour at room temperature in 5\% nonfat milk and Tris-buffered saline containing $0.1 \%$ Tween-20 solution (TBST). Membranes were then incubated with primary antibodies against claudin-5 (1:1000, Invitrogen, 34-1600), Bmal-1 (1:1000, Bethyl Laboratories Inc., A302$616 \mathrm{~A})$, and $\beta$-actin (1:5000, Abcam, ab8227) overnight at $4^{\circ} \mathrm{C}$; washed twice for 5 minutes each in TBST; and incubated with HRP-conjugated goat anti-rabbit secondary antibody (1:5000, MilliporeSigma, A6154) for 2 hours at room temperature. After four 10-minute washes in TBST, protein bands were visualized using enhanced chemiluminescence (Advansta) with an image analyzer (LI-COR C-DiGit scanner). Densitometry was performed using ImageJ with protein bands of interest normalized to the loading control $\beta$-actin.

qPCR analysis. Reverse transcription PCR (RT-PCR) was carried out using a SensiFAST SYBR Hi-ROX Kit (Bioline) according to the manufacturer's instructions. PCR was performed in a Step-One Plus Real-Time PCR instrument (Applied Biosystems). RT-PCR conditions were as follows: $95^{\circ} \mathrm{C}$ for 10 minutes, 37 cycles of $95^{\circ} \mathrm{C}$ for 15 seconds, and $60^{\circ} \mathrm{C}$ for 30 seconds. A melt curve stage was added: $95^{\circ} \mathrm{C}$ for 15 seconds, $60^{\circ} \mathrm{C}$ for 1 minute, and $95^{\circ} \mathrm{C}$ for 15 seconds. The comparative $\Delta \Delta \mathrm{CT}$ method was used to quantify changes in mRNA levels between treatment groups. The following primers were designed using Primer3: claudin-5 forward, 5'-TTTCTTCTATGCGCAGTTGG-3'; reverse, 5'-GCAGTTTGGTGCCTACTTCA-3'; occludin forward, 5'-ACAGTCCAATGGCCTACTCC-3'; reverse, 5'-ACTTCAGGCACCAGAGGTGT-3'; ZO-1 forward, 5'-CCACCTCTGTCCAGCTCTTC-3'; reverse, 5'-CACCGGAGTGATGGTTTTCT-3'; Bmal-1 forward, 5'-ATCAGCGACTTCATGTCTCC-3'; reverse, 5'-CTCCCTTGCATTCTTGATCC-3'; and $\beta$-actin forward, 5'-TCACCCACACTGTGCCCATCTACGA-3'; reverse, 5'-CAGCGGAACCGCTCATTGCCAATGG-3'

Biotin permeability assay. Mice were injected i.v. through the tail vein with $2 \mathrm{mg} / \mathrm{mL}$ EZ-Link Sulfo-NHS-Biotin $(600 \mathrm{Da})$. Eyes were enucleated 5 minutes after biotin injection and immersion fixed in $4 \%$ paraformaldehyde (PFA, $\mathrm{pH} 7.4$ ) overnight at $4^{\circ} \mathrm{C}$. Eyes were cryoprotected by sequential incubations in $10 \%, 20 \%$, and 30\% sucrose and frozen in OCT freezing medium (VWR). Mouse retinal cryosections were incubated with Cy3-conjugated streptavidin (1:200) overnight at $4^{\circ} \mathrm{C}$ to label Sulfo-NHS-Biotin. Biotin leakage was visualized with a Zeiss LSM 710 confocal laser scanning microscope. Image analysis was performed in ImageJ by drawing regions of interest outside blood vessels and measuring the mean pixel intensity.

MRI. iBRB integrity was assessed in vivo via MRI, using a dedicated small-rodent 7-T MRI system located at TCD (https://www.tcd.ie/Neuroscience/technologies/neuroimaging.php). Anesthetized mice were physiologically monitored (ECG, respiration, and temperature) and placed on an MRI-compatible support cradle, with a built-in system for maintaining the animal's body temperature at $37^{\circ} \mathrm{C}$. The cradle was then positioned within the MRI scanner. Accurate positioning was ensured by acquiring an initial rapid pilot image, which was then used to ensure the correct geometry was scanned in 
all subsequent MRI experiments. T1-weighted magnetic resonance images (resolution, $0.156 \times 0.156$ $\times 5 \mathrm{~mm}^{3}$; field of view: $20 \times 20 \times 17.9 \mathrm{~mm}^{3}$; matrix: $128 \times 128 \times 30$; TR/TE: $500 / 2.7 \mathrm{~ms}$; flip angle: $30^{\circ}$; number of averages: 3; acquisition time: 2 minutes, 24 seconds; repetitions: 12 ) were acquired following administration of $100 \mu \mathrm{L}$ of a 1:3 dilution of gadolinium diethylene-triamine penta-acetic acid (Gd-DTPA), administered via the tail vein. A quantitative assessment of iBRB integrity was also made using DCE-MRI, in which the passage of Gd-DTPA from the vasculature into the tissue was monitored over a period of 10 minutes after injection.

AAV2/9 design and generation. shRNAs designed to target transcripts derived from mouse or African green monkey claudin-5 were incorporated into AAV-2/9 vectors. Claudin-5 shRNA was subcloned into the pSingle-tTS-shRNA (Clontech) vector, which uses a doxycycline-inducible promoter. The plasmid incorporating the inducible system with claudin-5 shRNA was digested with BsrBi and BsrGI and ligated into the Not1 site of the plasmid pAAV-MCS so as to incorporate left and right AAV inverted terminal repeats. AAV-2/9 was then generated using a triple transfection system in a stably transfected HEK293 cell line for the generation of high-titer viruses (Vector BioLabs).

Cldn5-knockdown mouse generation. Mice were generated by standard blastocyst injection (32) at Charles Rivers Laboratory in collaboration with Mirimus Inc using their strict VAF/Elite health standards. A doxycycline-inducible claudin-5 shRNA was knocked in at the Colla1 locus on chromosome 11. Additionally, a CAG-lox-stop-lox-rtTA3-IRESmKate2 (CLR3K) allele was knocked in at the Rosa26 locus on chromosome 6. Mice homozygous for the claudin-5 shRNA-containing gene and the reverse tetracycline-controlled transactivator gene were crossed to Tie2-Cre-expressing animals for endothelial-specific expression of claudin-5 shRNA. Cre-negative littermate mice were used as controls. Doxycycline was administered to the drinking water ( $2 \mathrm{mg} / \mathrm{mL}$ in $2 \%$ sucrose solution) to activate shRNA expression and replenished 3 times a week.

FFA and OCT analysis. FFA and OCT were performed on mice using a Heidelberg SPECTRALIS OCT (Heidelberg Engineering). Pupils were dilated with 1\% tropicamide and 2.5\% phenylephrine and mice anesthetized using a mixture of ketamine and domitor. Mice were intraperitoneally injected with sodium fluorescein (2 $\mathrm{mg} / \mathrm{mL})$ at the same volume/weight $(100 \mu \mathrm{L} / 20 \mathrm{~g})$ to visualize the blood vessels. For the size selectivity study, mice were injected with the same volume/weight of FITC-Dextran FD4 (MilliporeSigma, $2 \mathrm{mg} / \mathrm{mL}$ ) in a similar fashion to sodium fluorescein. FFA images were captured from 2 minutes to 10 minutes every 30 seconds. FFA and OCT images were captured with a $30^{\circ}$ angle of view. Heidelberg Eye Explorer (HEYEX) version 1.7.1.0 was used to capture images. For quantification of microvessel permeability, ImageJ was used for analysis.

RNAi experiment. Brain endothelial cells (bEnd.3) (ATCC) were transfected with 10 pmol NT or Bmal1 siRNA using Lipofectamine 2000 transfection reagent (Thermo Fisher Scientific) diluted in OptiMEM reduced serum medium (Thermo Fisher Scientific) according to the manufacturer's instructions. Forty-eight hours after transfection, medium was replaced with medium containing $50 \%$ fetal calf serum, and cells were serum shocked for 2 hours. Following serum shock, cells were incubated in serum-free medium, and protein lysates were collected every 4 hours for 28 hours.

Immunocytochemistry. Confluent bEnd.3 cells were washed twice in PBS and fixed with 4\% PFA for 10 minutes at room temperature. Following fixation, cells were washed twice with PBS and blocked/permeabilized with 5\% NGS and $0.05 \%$ Triton X-100 in PBS for 1 hour at room temperature. Cells were incubated in primary antibody against claudin-5 (Invitrogen, 34-1600) (1:100) overnight at $4^{\circ} \mathrm{C}$. Cells were washed 3 times with PBS and incubated with goat anti-rabbit Cy3 (Abcam, ab6939) (1:500) secondary antibody for 2 hours at room temperature, followed by 4 washes with PBS. Nuclei were stained with Hoechst 33258 $(1: 10,000)$. The slide was removed from the chamber and mounted with Aqua Poly/Mount (Polysciences, Inc) before visualization by confocal laser scanning microscopy.

TEER. TEER measurements were performed on cells grown on 6.5-mm-diameter, 0.4- $\mu$ m-pore, polyester membrane HTS Transwell inserts (Corning Costar). TEER values were measured using an EVOM resistance meter (MilliporeSigma) fitted with "chopstick" electrodes. Before measurements, the apical and basolateral chambers were replaced with fresh medium. TEER values were recorded in triplicate, and the average value of a no-cell compartment was subtracted from the average of the triplicate measurements. TEER values were expressed as ohm $\times \mathrm{cm}^{2}\left(\Omega \times \mathrm{cm}^{2}\right)$.

High-fat diet and HCD study. C57BL/6J mice were fed ad libitum an HCD (Envigo, TD.88051).

Nonhuman primate study. Six monkeys were used for the FFA study, while 3 monkeys were used for the subretinal AAV study. Pupils were dilated with $1 \%$ tropicamide and $2.5 \%$ phenylephrine hydrochloride drops. 
Subretinal injections of AAV expressing claudin-5 shRNA under the control of a doxycycline-inducible promoter were performed under a surgical microscope in 3 African green monkeys under aseptic conditions under ketamine/xylazine anesthesia $(5.0 \mathrm{mg} / \mathrm{kg}$ ketamine [Fort Dodge] $/ 1.0 \mathrm{mg} / \mathrm{kg}$ xylazine [Lloyd Lab] administered intramuscularly in a sterilely mixed cocktail to effect). After placement of a lid speculum, a radial conjunctival peritomy incision was made in the superior temporal quadrant and a pars plana sclerotomy site marked on the sclera. A sclerotomy incision was made at the marked location using a 25-gauge MVR knife. After an anterior chamber paracentesis by 30-gauge needle at the limbal cornea, and application of a sterile hydroxypropyl methylcellulose contact lens coupling solution, a Machemer fundus contact lens was placed on the cornea. A 30-gauge anterior chamber cannula, used as a subretinal injector, was then introduced through the superior temporal sclerotomy into the vitreous cavity under the operating microscope. The cannula was then maneuvered so that it was apposed to the posterior temporal retinal surface at an oblique angle. The cannula was advanced to catch a fold of retina on the cannula tip and then advanced further to pierce the retina, avoiding injury to the underlying RPE and choroid. AAV was then injected in a small volume to confirm position and patency of the cannula tip. Once subretinal positioning was confirmed, the total target volume of $100 \mu \mathrm{L}$ of CLDN5 AAV-2/9 or AAV9shAnti-Luc control vector was injected, with monitoring of the injection under direct visualization through the microscope. The cannula was then removed from the retina and scleral insertion site. Tobramycin and dexamethasone ointment (Tobradex, Alcon) was administered topically following the injection procedure.

Nonhuman primate FFA. Fundus color photography and FFA were performed on 6 ketamine/xylazineanesthetized animals using a retinal camera (Topcon TRC 50X, Topcon America Corp) with Canon D4 digital imaging hardware and New Vision Fundus Image Analysis System software. FFA in the left eye was preceded by angiography in the right eye by 6 hours to allow adequate time for washout of fluorescein between image series. Color and red-free images were collected from each eye before infusion of $0.1 \mathrm{~mL} / \mathrm{kg}$ $10 \%$ sodium fluorescein into the saphenous vein. A rapid series of angiogram images were collected from the posterior pole immediately after completion of the infusion for the first minute and then at 2,3 , and 6 minutes. A single angiogram was also taken from the contralateral eye after collection of the 6-minute angiogram image to ensure intereye consistency in fluorescein levels in the optic nerve head and normal vasculature. Color and red-free photographs and fluorescein angiograms were collected from each animal at 14 days after injection.

Nonhuman primate OCT examinations. OCT was performed using a Heidelberg Spectralis OCT Plus with eye tracking and HEYEX image capture and analysis software. Images were acquired from all the eyes at baseline, immediately after injection, and at 14 days (immediately before sacrifice). At all time points an overall volume scan of the entire macula was performed at a dense scan interval, encompassing the subretinal injection site where applicable.

Nonhuman primate termination. On day 42 after injection, monkeys were euthanized by pentobarbital overdose while under ketamine sedation and globes were enucleated. Globes were marked at the 12'00 position with indelible ink before enucleation. Extraocular tissues were trimmed and the globes were placed into 4\% PFA fixative without further manipulation. Following 24-hour fixation the globes were maintained in PBS with $0.05 \%$ sodium azide until sectioning for histopathology and immunohistochemistry.

Human FFA study. Fifteen healthy volunteers were recruited from Dublin, Ireland, and informed consent was obtained from all participants. Fundus color photography was performed using a retinal camera (Topcon TRC 50X, Topcon America Corp) with Canon D4 digital imaging hardware, and FFA was performed using a Heidelberg Spectralis OCT (Heidelberg Engineering). Pupils were dilated with $1 \%$ tropicamide and $2.5 \%$ phenylephrine. Sodium fluorescein $(500 \mu \mathrm{g})$, followed by a $5-\mathrm{mL}$ flush of $0.9 \%$ sodium chloride, was injected via a peripheral cannulation site, to visualize the blood vessels of the posterior pole. Color and red-free images were collected from each eye before injection of sodium fluorescein. FFA images of the posterior pole were captured every 15 seconds from completion of the infusion to 10 minutes. The images were captured with a $30^{\circ}$ angle of view in the left eye of all participants. HEYEX version 1.7.1.0 was used to capture images. Fundus images were independently reviewed by a consultant ophthalmologist, and ImageJ analysis was used for quantification of microvessel permeability.

Human donor eye RNA analysis. Peripheral nasal retina and central retina, including the macula, were isolated from human donor eyes within an average of approximately 11 hours and frozen separately $(n=28$ donor eyes). RNA was isolated using the RNeasy kit (Qiagen). 
Circadian open-source data. Initial data outlining claudin-5 transcript cycling outlined in Figure 1, A and B, were collated from open-source data available on http://cirgrdb.biols.ac.cn/ and referenced in Xianfeng et al. (33).

Statistics. Statistical analysis was performed using a 2-tailed Student's $t$ test, with significance represented by a $P$ value of less than or equal to 0.05 when 2 individual experimental groups were being analyzed. For multiple comparisons, 1-way ANOVA was used with Bonferroni's post hoc test and significance represented by a $P$ value of less than or equal to 0.05 . Error bars represent SEM.

Study approval. All ethical approvals for animal and human experiments outlined in this study were obtained from relevant local and national competent authorities before the initiation of the studies. Studies outlined in animals and humans were reviewed and approved by an appropriate institutional review board. The TCD Animal Research Ethics Committee approved all animal mouse studies. The St Kitts Biomedical Research Foundation approved all nonhuman primate studies. The Royal Victoria Eye and Ear Hospital Research Foundation ethics committee approved the human studies. Informed consent was obtained from each participant in the human FFA study.

\section{Author contributions}

NH conceived, designed, and performed experiments and analyzed data. LC performed FFA analysis. AH conducted FFA analysis in human subjects. C Greene generated claudin-5-knockdown mice. FS performed qPCR on human donor eyes. EO performed OCT and FFA analysis. EF performed Western blot and qPCR analysis. ST performed histological analysis. PFK assisted with human FFA analysis and performed human FFA analysis. MMH genotyped mice. AMC assisted in design and implementation of circadian studies. $\mathrm{ED}, \mathrm{AB}$, and SL performed nonhuman primate study analysis. MSL designed and implemented nonhuman primate studies. MTC designed and implemented the human FFA study. C Grimm designed human donor eye tissue experiments. PH designed and managed the study. SLD conceived experiments and wrote the paper. MC conceived experiments and wrote the paper.

\section{Acknowledgments}

This work was supported by grants from Science Foundation Ireland (SFI) (12/YI/B2614 and 11/ PI/1080), the Health Research Board of Ireland, the BrightFocus Foundation, the Irish Research Council, Enterprise Ireland, and The Research Foundation at the Royal Victoria Eye and Ear Hospital. The Campbell lab at TCD is also supported by an SFI Centres grant supported in part by a research grant from SFI under grant number 16/RC/3948 and cofunded under the European Regional Development Fund by FutureNeuro industry partners. The authors thank Joshua Dunaief and Hannah Schultz for assistance with histopathology. We thank Caroline Woods and Charles Murray for animal husbandry.

Address correspondence to: Matthew Campbell, Smurfit Institute of Genetics, Lincoln Place Gate, Trinity College Dublin, Dublin 2, Ireland. Phone: 353.1.8961482; Email: Matthew.Campbell@tcd.ie.

1. Colijn JM, et al. Prevalence of age-related macular degeneration in Europe: the past and the future. Ophthalmology. 2017;124(12):1753-1763.

2. Comparison of Age-related Macular Degeneration Treatments Trials (CATT) Research Group, et al. Five-year outcomes with anti-vascular endothelial growth factor treatment of neovascular age-related macular degeneration: the comparison of age-related macular degeneration treatments trials. Ophthalmology. 2016;123(8):1751-1761.

3. Fleckenstein $\mathrm{M}$, et al. The progression of geographic atrophy secondary to age-related macular degeneration. Ophthalmology. 2018;125(3):369-390.

4. Reynolds R, Rosner B, Seddon JM. Dietary omega-3 fatty acids, other fat intake, genetic susceptibility, and progression to incident geographic atrophy. Ophthalmology. 2013;120(5):1020-1028.

5. Crabb JW, et al. Drusen proteome analysis: an approach to the etiology of age-related macular degeneration. Proc Natl Acad Sci US A. 2002;99(23):14682-14687.

6. Wang L, et al. Abundant lipid and protein components of drusen. PLoS One. 2010;5(4):e10329.

7. Curcio CA. Antecedents of soft drusen, the specific deposits of age-related macular degeneration, in the biology of human macula. Invest Ophthalmol Vis Sci. 2018;59(4):AMD182-AMD194.

8. LaVail MM. Rod outer segment disk shedding in rat retina: relationship to cyclic lighting. Science. 1976;194(4269):1071-1074

9. Boyle D, Tien LF, Cooper NG, Shepherd V, McLaughlin BJ. A mannose receptor is involved in retinal phagocytosis. Invest Ophthalmol Vis Sci. 1991;32(5):1464-1470.

10. Kim JY, et al. Noncanonical autophagy promotes the visual cycle. Cell. 2013;154(2):365-376.

11. Yao J, et al. Inhibiting autophagy reduces retinal degeneration caused by protein misfolding. Autophagy. 2018;14(7):1226-1238. 
12. Barondes M, Pauleikhoff D, Chisholm IC, Minassian D, Bird AC. Bilaterality of drusen. Br J Ophthalmol. 1990;74(3):180-182.

13. Mann SS, et al. The symmetry of phenotype between eyes of patients with early and late bilateral age-related macular degeneration (AMD). Graefes Arch Clin Exp Ophthalmol. 2011;249(2):209-214.

14. Campbell M, Humphries P. The blood-retina barrier. In: Cheng CY, ed. Biology and Regulation of Blood-Tissue Barriers. Advances in Experimental Medicine and Biology. New York, New York, USA; Springer; 2013:70-84

15. Díaz-Coránguez M, Ramos C, Antonetti DA. The inner blood-retinal barrier: cellular basis and development. Vision Res. 2017;139:123-137.

16. Wolburg H, Lippoldt A. Tight junctions of the blood-brain barrier: development, composition and regulation. Vascul Pharmacol. 2002;38(6):323-337.

17. Bazzoni G, Dejana E. Endothelial cell-to-cell junctions: molecular organization and role in vascular homeostasis. Physiol Rev. 2004;84(3):869-901

18. Tsukita S, Furuse M, Itoh M. Multifunctional strands in tight junctions. Nat Rev Mol Cell Biol. 2001;2(4):285-293.

19. Vorbrodt AW, Dobrogowska DH. Molecular anatomy of intercellular junctions in brain endothelial and epithelial barriers: electron microscopist's view. Brain Res Brain Res Rev. 2003;42(3):221-242.

20. Daneman R, Zhou L, Agalliu D, Cahoy JD, Kaushal A, Barres BA. The mouse blood-brain barrier transcriptome: a new resource for understanding the development and function of brain endothelial cells. PLoS One. 2010;5(10):e13741.

21. Greene C, et al. Dose-dependent expression of claudin-5 is a modifying factor in schizophrenia. Mol Psychiatry. 2018;23(11):2156-2166.

22. Dierickx P, Van Laake LW, Geijsen N. Circadian clocks: from stem cells to tissue homeostasis and regeneration. EMBO Rep. 2018;19(1):18-28

23. Balsalobre A, Damiola F, Schibler U. A serum shock induces circadian gene expression in mammalian tissue culture cells. Cell. 1998;93(6):929-937.

24. Toomey CB, Kelly U, Saban DR, Bowes Rickman C. Regulation of age-related macular degeneration-like pathology by complement factor H. Proc Natl Acad Sci U S A. 2015;112(23):E3040-E3049.

25. Wyse CA, Coogan AN. Impact of aging on diurnal expression patterns of CLOCK and BMAL1 in the mouse brain. Brain Res. 2010;1337:21-31.

26. Ando H, et al. Influence of age on clock gene expression in peripheral blood cells of healthy women. J Gerontol A Biol Sci Med Sci. 2010;65(1):9-13.

27. Kondratov RV, Kondratova AA, Gorbacheva VY, Vykhovanets OV, Antoch MP. Early aging and age-related pathologies in mice deficient in BMAL1, the core component of the circadian clock. Genes Dev. 2006;20(14):1868-1873.

28. Clemons TE, Milton RC, Klein R, Seddon JM, Ferris FL, Age-Related Eye Disease Study Research Group. Risk factors for the incidence of advanced age-related macular degeneration in the Age-Related Eye Disease Study (AREDS) AREDS report no. 19 Ophthalmology. 2005;112(4):533-539.

29. van Romunde SH, Polito A, Bertazzi L, Guerriero M, Pertile G. Long-term results of full macular translocation for choroidal neovascularization in age-related macular degeneration. Ophthalmology. 2015;122(7):1366-1374.

30. Takeuchi K, Kachi S, Iwata E, Ishikawa K, Terasaki H. Visual function 5 years or more after macular translocation surgery for myopic choroidal neovascularisation and age-related macular degeneration. Eye (Lond). 2012;26(1):51-60.

31. Ghorbanian S, Jaulim A, Chatziralli IP. Diagnosis and treatment of coats' disease: a review of the literature. Ophthalmologica. 2012;227(4):175-182.

32. Behringer R, Vintersten Nagy K, Nagy A. Manipulating the Mouse Embryo: A Laboratory Manual, Fourth Edition. Plainview, New York, USA: Cold Spring Harbor Laboratory Press; 2014

33. Li X, et al. CirGRDB: a database for the genome-wide deciphering circadian genes and regulators. Nucleic Acids Res. 2018:46(D1):D64-D70. 